\title{
A Greenhouse Project toward Smart Agriculture
}

\author{
Kazuo Ishii ${ }^{1}$, Yuya Nishida ${ }^{1}$, Shinsuke Yasukawa ${ }^{1}$, Kanako Shirahashi ${ }^{1}$, \\ Yasunori Takemura², Takayuki Matsuo ${ }^{3}$ \\ ${ }^{I}$ Center for Socio-Robotic Synthesis, Kyushu Institute of Technology, 2-4, Hibkinino, Wakamatsu \\ Kitakyushu, Fukuoka 808-0196, Japan \\ ${ }^{2}$ Department of Engineering, Nishinippon Institute of Technology, 1-11 Aratsu, Kanda, \\ Miyakogun, Fukuoka 800-0394, Japan \\ ${ }^{3}$ National Institute Technology, Kitakyushu College \\ 2-20-1, Shii, Kokuraminami-ku, kitakyushu-shi, Fukuoka, Japan
}

E-mail: ishii@brain.kyutechg.ac.jp,matsuo@kct.ac.jp,takemura@nishitech.ac.jp,sonoda@nishitech.ac.jp, y-nishida@brain.kyutech.ac.jp,s-yasukawa@brain.kyutech.ac.j

\begin{abstract}
In the future estimation of 2050 , the food demand increases $70 \%$ and the production decreases $15 \%$ caused by global warming, and farmer population be 1/5 of current workers. Until 2050, we need an agricultural system of twice production with the same farmland area and 5 times effective operations. To realize the sustainable society, smart agriculture including robot technology, AI, IoT is one of the solutions for food issues. We have been working for a greenhouse project under enPiT-everi for implementation of robotic, AI and IoT technologies to agriculture. In this paper, we introduce the project and robotic applications.
\end{abstract}

Keywords: tomato harvesting, agriculture robot, smart agricultu

re

\section{Introduction}

Due to the frequent occurrence of intense heat and heavy rain, the harsh global environment caused by global warming, world food production will decrease by $15 \%$ to 2.9 billion tons in 2050[1]. Meanwhile, the world population has increased to her 8.6 billion and food demand is expected to be a serious food shortage that will reach 5.8 billion tones [2]. Because of the progress of desertification and global warming, rapid expansion of excellent agricultural land cannot be expected. Therefore, in order to eliminate food shortages, it is necessary to obtain twice the yield of the same area as before. In addition to the intense heat and heavy rain limiting people's outdoor activities, it is estimated that the number of farmers in Japan will decrease sharply to one-fifth due to the declining birthrate and aging population [3], resulting in a serious labor shortage. It is expected that the production will not be able to meet global food demand. To realize the sustainable society, smart agriculture including robot technology, AI, IoT is one of the solutions for food issues.

On the other hand, the Ministry of Education, Culture, Sports, Science and Technology of Japan (MEXT) planned a program to form practical education networks for industry-academia collaboration in order to strengthen the function of developing human resources 
who can solve specific problems in society by making advanced use of information technology, and practice problem-based learning "enPiT" [4] with the aim of promoting various education and spreading it nationwide. Our university, Kyushu Institute of Technology, proposed an educational program named "enPiT-everi" (Evolving and Empowering Regional Industries) [5] to grow up small and middle size companies in Kyushu region together with Kitakyushu-city university and so on. We have been working for a greenhouse project for evaluation of robotic, AI and IoT technologies as a part of enPiT-everi. In this paper, we introduce the project and robotic applications for agriculture.

\section{Smart Agriculture}

Smart agriculture is a new style of agriculture that utilizes robot technology and information and communication technology (ICT) to promote labor saving, precision, and high-quality production. In Japanese agriculture fields, there are still many tasks that depend on manpower and can only be done by skilled workers. The labor saving, securing manpower, and reducing the burden in Japanese agriculture are important issues. By utilizing smart agriculture that makes full use of advanced technology, it is possible to overcome the issues in agricultural work, secure new farmers, and improve cultivation technology.

(1) Labor saving and labor reduction of agricultural work Japanese agriculture is facing a serious labor shortage due to the aging of individual farmers. It is required to support such hardships in Japanese agriculture by utilizing ICT.

\section{Succession of agricultural technology}

The second issue is the transfer of cultivation technology to new farmers. In the old system, the knowledge is transferred in families or small groups. The shortage of human resources makes difficult to inherit expert knowledge in agriculture in the old fashion.

\section{Improvement of food self-sufficiency}

Japan's food self-sufficiency rate (calorie basis) was about $40 \%$ in FY2018, and imports far exceed domestic production. In order to increase the yield and increase the self-sufficiency rate in the face of the above-mentioned shortage of human resources, automation by IoT, AI and robots is indispensable for reliably growing agricultural products with a small number of workers.

\section{3. enPiT-Pro and enPiT-everi}

The Ministry of Education, Culture, Sports, Science and Technology of Japan (MEXT) planned a program to form practical education networks for industry-academia collaboration in order to strengthen the function of developing human resources who can solve specific problems in society by making advanced use of information technology, and practice problem-based learning "enPiT" with the aim of promoting various education and spreading it nationwide. From 2017, as enPiT-Pro, MEXT supports the universities to develop and implement a systematic, advanced and short-term practical education program for working adults, mainly in the field of information science and technology, in collaboration with industry and multiple universities. By disseminating the program widely throughout Japan, MEXT also supports graduate school to reform efforts aimed at contributing to strengthening the re-learning function of working adults in the entire field in Japan.

Our university, Kyushu Institute of Technology,

proposed an educational program named "enPiTeveri” (Evolving and Empowering Regional Industries) to grow up small and middle size companies in Kyushu region together with Kitakyushu-city univ., Kumamoto univ., Miyazaki univ. and Hiroshima-city univ.. In the enPiT-everi program, we provide practical educational programs for working people in the Kyushu and Chugoku regions to acquire new technologies such as artificial intelligence and robot technology. The educational themes are specialized for manufacturing industry, automobile industry, nursing care industry, agriculture, forestry and livestock industry, tourism industry. The enPiT-everi develops human resources who can promote the introduction to companies such as IoT, AI and robots. One of the educational themes is the implementation of IoT for agriculture, and we have been developing the experimental greenhouse with IoT and robotic technologies.

\section{Greenhouse project in enPiT-everi}

The greenhouse developed for smart agriculture is shown in Fig.1. The house size is $10 \mathrm{~m} \times 20 \mathrm{~m}$ and the half 
area is designed for cultivation with soil and the other is for hydroponics.

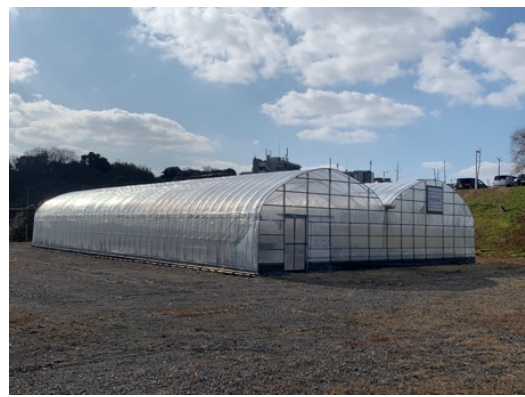

(a) Overview of the house
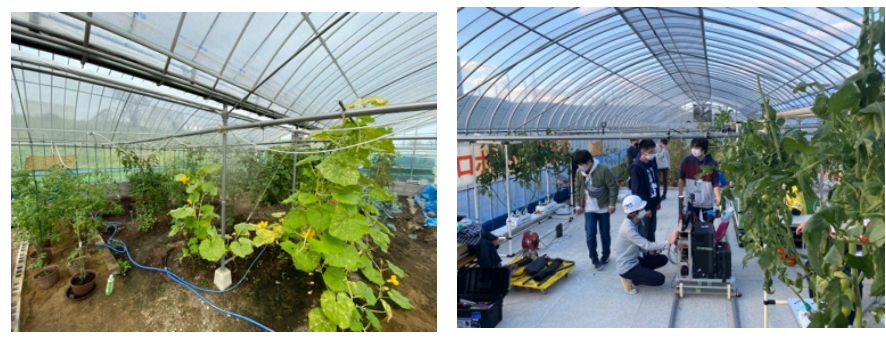

(b) Inside the house

Fig.1 The experimental greenhouse for agriculture IoT exercise.

Currently, as agricultural IoT Sensors, temperature, moisture data, $\mathrm{pH}$ regular measurement of soil, regular measurement of temperature, humidity and illuminance in the greenhouse, photography of plants, have been implemented. As actuators for control air condition and soil condition, opening and closing the greenhouse walls, spraying mist, water supply control by solenoid valves, water circular system in hydroponics, the system for purification of rainwater and control of nutrients are under implementation.

Using the experimental house, we will encounter to various problems in agriculture. For example, recognition of green worms imitating the leaf vein, disease of plants, harvesting and so on. Transmitting data to cloud data base is also one research topics with limited communication like Sigfox, Lora, etc. The detail of exercise for Agricultural IoT course is presented in the paper [5] in this conference. Also the TomatoHarvesting-Robot Competition was held in the greenhouse from 7 th competition in 2020 [6].
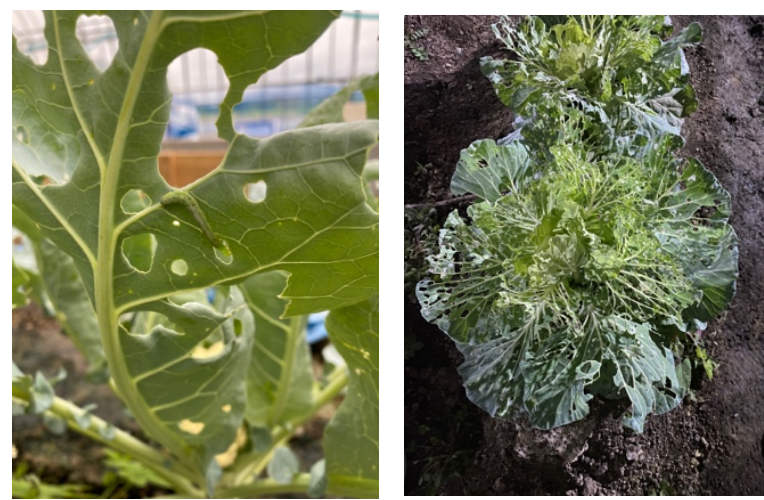

(a) green worms imitating the leaf vein
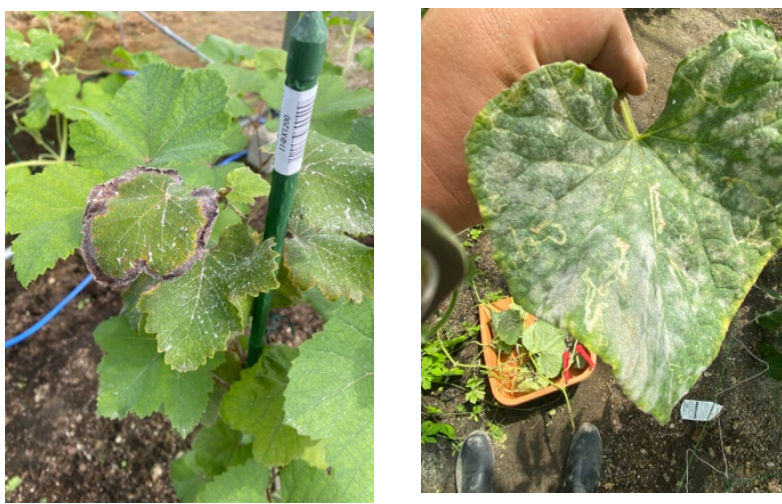

(b) Disease detection

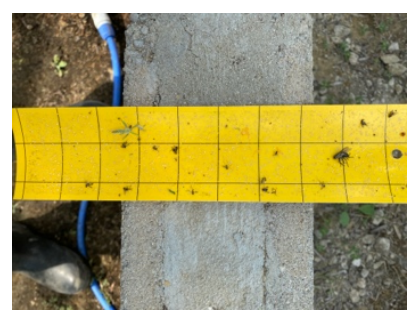

(c) Insect counting

Fig. 2 Research issues related to the greenhouse. 


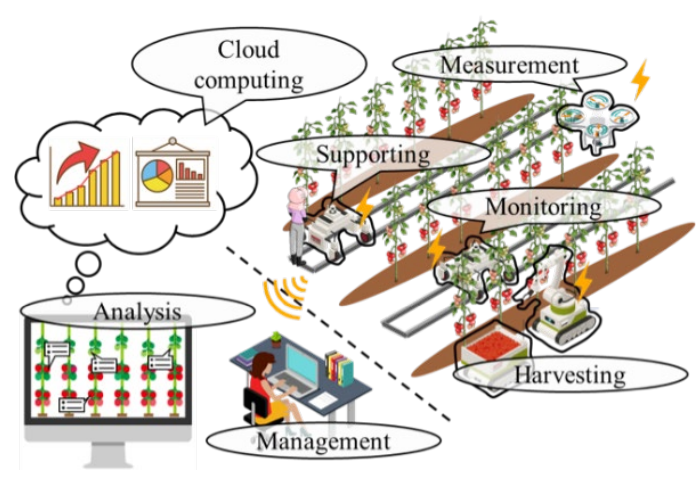

Fig. 3 The future concept of the greenhouse project.

\section{Summary}

We have been working for experimental greenhouse under enPiT-everi educational program. The agricultural IoT devices are implemented toward smart agriculture. Future vision of the greenhouse is shown in Fig. 3. The house system will include IoT sensors and actuators to control environment, robots for monitoring, harvesting, and transportation. Using the greenhouse, the new research topics with actual demands will come up toward smart agriculture.

\section{References}

1. https://www.maff.go.jp/j/tokei/sihyo/index.html

2. https://www.maff.go.jp/primaff/seika/attach/pdf/200403 2019_01.pdf

3. https://www.maff.go.jp/primaff/seika/fsc/faccess/a_map. html

4. http://www.mext.go.jp/a_menu/koutou/kaikaku/enpit/ind ex.htm

5. https://www.enpit-everi.jp

6. Y. Takemura et al., Smart Agriculture IoT Education Course in enPiT-everi (Education Network for Practical Information Technologies - Evolving and Empowering Regional Industries), Proc of ICAROB2021, to be appear, 2021.

7. K. Ishii et al., Tomato-Harvesting-Robot Competition Towards Smart Agriculture, Proc of ICAROB2021, to be appear, 2021.

8. T. Matsuo, et al., Tomato-Harvesting Robot Competition: Aims and Developed Robot of 6th Competition, Proc of ICAROB2021, to be appear, 2021 